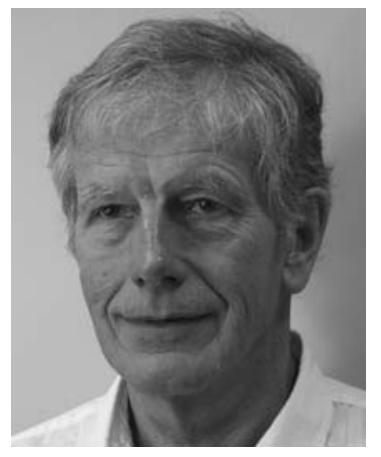

\title{
A Conversation with Ueli Schibler
}

\author{
INTERVIEWER: EyTAN ZLOTORYNSKI
}

Senior Editor, Nature Reviews: Molecular Cell Biology

Ueli Schibler is Professor in the Department of Molecular Biology at the University of Geneva.

Eytan Zlotorynski: You study the regulation of the circadian clock. Perhaps you could describe phase entrainment, the process by which the circadian clock is regulated throughout all the cells of an organism.

Dr. Schibler: Throughout the four billion years of life on Earth, organisms have been exposed to changes in the light/dark cycle, what we call the photoperiod. This changes the entire behavior of most organisms, at least those that are sensitive to light. We have a rest/activity cycle. Some, like humans, are more active during the day whereas laboratory rodents are more active during the night and sleep during the day. We can see whether it's light or dark, so why couldn't we just adapt our physiology that way? The answer is that the clock lets organisms anticipate time. A mouse is inactive during the day but, without an endogenous clock it would never know when it should become active. It can't check every 10 minutes to see if it's dark. It would never sleep, and maybe the snakes that are awake during the day would eat it. It's an advantage to be able to anticipate time. For example, when you eat, you intoxicate yourself, particularly with plant components. Most people believe that plants are much healthier than meat, but if you really investigate the problem, you'll come out with a different answer. It's good to have the appropriate enzymes already at high levels before you eat so you are ready to react to the insult. The same can be said for most of our physiology.

More than two decades ago, scientists discovered that we have a clock located in the hypothalamus of the brain in a small structure, called the suprachiasmatic nucleus $(\mathrm{SCN})$, and the SCN gets photic information from the retina through the retinohypothalamic tract in the form of electrical activity via synaptic transmissions. Daily exposure to light can reset the clock to the proper phase, but the SCN clock runs, no matter what. You can keep mice for generations in a dark room. They don't mind because they don't like light, and they'll stay rhythmic throughout their lifetimes, even through generations.

Nineteen ninety-seven was a big year for mammalian clock genes. Joe Takahashi, who's now at the University of Texas-Southwestern Medical Center, discovered the first clock gene using forward genetic techniques. Then the genomics studies came along, so you could just look up the Drosophila clock genes and see if there were similar genes in humans or mice. Now there are at least 20 clock genes that we know of that are important, although we still don't understand how these clock genes generate a rhythm of approximately 24 hours. We do know that there's a feedback loop, and when you remove certain genes, the organism doesn't have a clock anymore. Several labs, including ours, have found that clock gene expression goes through regular cycles in every tissue in the body.

The question then becomes whether the cells need their own internal clocks, or if the main clock in the brain just sends out hormones that drive these rhythms. A postdoc in my laboratory demonstrated that clocks are actually a cell-autonomous property. You can take cultured cells and synchronize their clocks, for example, by a serum shock or by glucocorticoid hormones. You don't need a cellular network; a single cell can do it. This is how we discovered these peripheral clocks, and then other people like Menaker and Takahashi and others took tissue samples and showed that they also have clocks. Every one of our 30 trillion cells has its own clock.

Eytan Zlotorynski: To study the circadian clock in live animals, you devised a real-time bioluminescence recorder of circadian clock gene expression. Could you explain how this device works?

Dr. Schibler: First of all, to study phase shifting in peripheral organs you have to take tissue samples at regular intervals over prolonged periods of time, sometimes days or weeks. It would be cruel to have to kill multiple mice of different genetic backgrounds every $4 \mathrm{~h}$ during many days. This would amount to hundreds of mice, just to be able to extract RNA and proteins to measure. Second, the resolution would be very poor because there are intraindividual differences, and you only have one point every $4 \mathrm{~h}$. Instead, we engineered an apparatus with some 
very good mechanical engineers in our department and the help of two photonics physicists. If the mice express inserted reporter transgene encoding a firefly enzyme under the control of circadian regulatory elements, we can capture the photons that come out of their body and count them. The mice are in a completely lightproof cage, and the photons are guided toward the surface of a photomultiplier tube. This is extremely sensitive and allows us to measure gene expression over prolonged time periods because we can add luciferin, the substrate for the enzyme, to the drinking water.

\section{Eytan Zlotorynski: What is its sensitivity?}

Dr. Schibler: We haven't determined the lower limit. If you put these reporter genes into liver cells using adenoviral vectors, in the best cases, we get one million photons per minute, and the machine background is 1000 . Depending on the gene, we can get a 40 - to 50 -fold oscillation. This is robust. Of course, we can't use this yet in the SCN, which only has 20,000 neurons, but I think we could go down by a factor of 50 and still get robust signals.

Eytan Zlotorynski: You've made some interesting discoveries using this device and other methods about how the circadian clock is regulated by external environmental cues and the transcriptional basis of that. Could you highlight your most important findings?

Dr. Schibler: The SCN is adjusted every day by the photoperiod to exactly $24 \mathrm{~h}$. Of course, there are clocks in tissues like the liver that don't get any light and, in addition, are light-insensitive. So there must be other ways to synchronize these peripheral clocks. This is important, because if you have billions of clocks but they're all ticking at a different rhythm, it doesn't help much for circadian physiology. Around 2000, we found that the dominant "time giver" - the field actually uses the German word "zeitgeber"- - for these peripheral clocks is the feeding/fasting rhythm. I actually believe that the fasting period is more important than feeding in setting the clocks.

Even in constant darkness, in the absence of photoperiod cues, the SCN won't change its own cycle to adapt to the feeding cycle. The SCN is the chief; it doesn't care about feeding, so even if you invert the feeding rhythms, it still keeps its own phase. In peripheral clocks, you can create unnatural conditions where you put the SCN phase in conflict with the feeding phase. We know there is a conflict because when we put animals on day feeding, which for these nocturnal animals is not normal, but then give food back ad libitum, it only takes 2 days to get the old phase because the SCN clock can reassert its dominance. If we do the opposite, have them on normal feeding and then put them on daytime feeding, it takes more than a week to adjust the phase because the SCN is trying to fight, to keep the other clocks ticking to its own phase. The SCN rhythm will try to reconcile the conflicting cycles, but ultimately it's not powerful enough.

With the photomultiplier apparatus, dubbed RT-Biolumicycler, we can actually measure this directly. We can lesion the brain of an animal to very precisely remove the SCN_-it's less than a cubic millimeter of tissue - and make them completely arrhythmic. Then you can make the liver and other organs rhythmic using feeding rhythms, and you can switch the feeding rhythms almost immediately if they don't have an SCN. If they do have an $\mathrm{SCN}$, it takes several days to weeks. That's how we know that the SCN is actually sending out other signals that are in conflict with feeding, when the feeding rhythm is inverted by the experimenter. The SCN uses a large number of signaling pathways, and some of them are quite indirect. Among the more direct signals are glucocorticoid hormones. We also just identified a proteinaceous signal in the blood that is used by the SCN. We don't know yet what that protein is. It's a signal protein at very low concentrations in the blood, but we do know that the downstream pathway that it regulates has to do with cytoskeletal actin dynamics.

Eytan Zlotorynski: So regardless of the SCN, if you have two contradicting environmental cues, is there a hierarchy? Is it a matter of strength or duration which one wins?

Dr. Schibler: We should be clear that imposing feeding rhythms is very artificial. We use these to measure phaseshifting kinetics and to measure the contribution of particular signaling pathways. Feeding rhythms are dominant over the SCN. The SCN cannot keep the phase of the periphery for long. It tries to do so, but it's unsuccessful. Even if you remove a signaling pathway depending on feeding-fasting cycles (e.g., poly(ADP) ribosylation of proteins), you will still adjust the phase to the feeding in the end. On the other hand, how quickly the peripheral clocks adjust their phase depends on the signaling pathway you have genetically disrupted: If it's governed by the SCN, then phase adaptation goes faster; if it's from the feeding, it takes longer.

Eytan Zlotorynski: What does this teach us about some of the more common medical problems that humans have that may have a circadian component to them?

Dr. Schibler: In the modern world humans are exposed to many factors perturbing the normal adjusting of clock to geophysical time. If you read your smartphone at night before you go to bed, it has a lot of blue and green light, which shifts the phase most efficiently. You also have rotation-shift workers: people who work at nights one week and during the days the next. It's fair to say, though, that the impact of rotation shiftwork on health is lower than I would have anticipated. It takes a long time for major effects to be seen, more than 10 years. If you travel frequently, you get jet lag all the time, but it's not going to kill you. Circadian rhythm perturbances are unpleasant because they cause sleep problems and, sometimes, digestive problems, but it's not world killer number one. I believe it is important to have your physiology rhythmic, but one shouldn't overexaggerate the consequences of perturbances if they don't last for decades. 


\section{$\$_{\text {CSH }}^{\infty}$ Cold Spring Harbor Symposia SYMPOSIA On Quantitative Biology}

\section{A Conversation with Ueli Schibler}

Cold Spring Harb Symp Quant Biol 2015 80: 344-345

Access the most recent version at doi:10.1101/sqb.2015.80.030122

\section{License}

Email Alerting Receive free email alerts when new articles cite this article - sign up in Service the box at the top right corner of the article or click here. 